\title{
EFEITOS DO TREINAMENTO SENSÓRIO MOTOR POR MEIO DE DISPOSITIVOS ECOEFICIENTES SOBRE A CAPACIDADE FUNCIONAL E EQUILÍBRIO EM IDOSOS: ENSAIO CLÍNICO CONTROLADO
}

\author{
Caroline Santos Gomes', Gabriela Manhães Braga Rangel', Maria Eduarda Gomes da Silva \\ Sant'Ana', Marília Aparecida Terra dos Santos ${ }^{1}$, Wanderson Lucas de Aguiar Fraga', Elizabeth \\ Viana Soares ${ }^{2}$, Sileno Martinho Silva Ribeiro Junior ${ }^{3}$
}

GOMES, C.S.; RANGEL, G.M.B.; SANT'ANA, M.A.T.S.; FRAGA, W.L.A.; SOARES, E.S.; JUNIOR, S.M.S.R. Efeitos do treinamento sensório motor por meio de dispositivos ecoeficientes sobre a capacidade funcional e equilíbrio em idosos: ensaio clínico controlado. Perspectivas Online: Biológicas \& Saúde. v. 8, n 28, p.42-58, 2018.

\section{RESUMO}

Introdução: Os idosos sofrem quedas recorrentes devido à diminuição do equilíbrio, força muscular e capacidade psicomotora. $\mathrm{O}$ treinamento sensório motor visa melhorar estas funções. Objetivo: Avaliar a capacidade funcional dos idosos utilizando testes validados e um programa de treinamento sensório motor sobre a capacidade funcional e equilíbrio. Metodologia: Foi realizado um ensaio clinico controlado com 10 idosas, com idade entre 60 e 80 anos divididos em 2 grupos, $3 \mathrm{x}$ semana durante 30 dias. $\mathrm{O}$ grupo Treinados foi composto por 6 idosas que realizaram o protocolo de intervenção, e o Grupo Não Treinados por 4 idosas que foram submetidas apenas a avaliação pré e pós através dos seguintes métodos: Escala de Berg, Teste de Alcance Funcional Anterior, Teste de Up Upgrade e Tempo
(TGUGM).Resultados: Foi observadouma melhora na Escala de Berg onde as idosas participantes do grupo Treinados ultrapassaram as pontuações, mantendo pontuação mínima de 49 . No TAF houve uma melhora no grupo Treinados com aumento de $3 \mathrm{~cm}$ no momento pós intervenção comparado ao momento pré. Já no TGUGM, a média alcançada foi de 7,8 \pm 1,3 no grupo Não Treinados e de 10,8 \pm 4,9 no grupo Treinados, obtendo uma qualidade do movimento.Conclusão: $\mathrm{O}$ treinamento sensório motor direcionado aos idosos promoveu um resultado positivo em relação à funcionalidade e equilíbrio estático e dinâmico, melhorando o desempenho das atividades de vida diária, diminuindo assim, fatores de risco preocupantes para quedas nesta população.

Palavras - Chave: Queda em Idosos, Equilíbrio, Funcionalidade. 


\begin{abstract}
Introduction: The elderly suffer recurrent falls due to decreased balance, muscular strength and psychomotor ability. Sensory motor training aims to improve these functions. Objective: To evaluate the functional capacity of the elderly using validated tests and a program of sensory motor training on functional capacity and balance. METHODS: A controlled clinical trial was conducted with 10 elderly women, aged 60 to 80 years divided into 2 groups, $3 \mathrm{x}$ week for 30 days. The Trained group consisted of 6 elderly women who underwent the intervention protocol, and the Non-trained Group by 4 elderly women who underwent only pre and post evaluation through the following methods: Berg Scale, Anterior Functional

(TGUGM) .Results: An improvement was observed in the Berg Scale where the elderly participants in the Trained group exceeded the scores, maintaining a minimum score of 49 . In the TAF there was an improvement in the group Trained with a $3 \mathrm{~cm}$ increase in the post intervention compared to the pre. In TGUGM, the mean reached was $7.8 \pm 1.3$ in the Untrained group and $10.8 \pm 4.9$ in the trained group, obtaining a quality of movement. Conclusion: Sensory motor training directed to the elderly promoted a positive result in relation to functionality and static and dynamic balance, improving the performance of activities of daily living, thus reducing worrying risk factors for falls in this population.
\end{abstract} Reach Test, Up Test Upgrading and Time

Keywords: Falling in the Elderly, Equilibrium, Functionality.

\footnotetext{
${ }^{1}$ Institutos Superiores de Ensino do CENSA -ISECENSA - Aluno (a) Voluntário (a) de Iniciação Científica PROVIC - Rua Salvador Correa, 139, Centro, Campos dos Goytacazes, RJ, CEP: 28035-310, Brasil.

2 Pesquisadora do Núcleo de Pesquisa em Fisioterapia Neuro Funcional - NUPEFIN/ISECENSA

${ }^{3}$ Pesquisador do Núcleo de Pesquisa em Fisioterapia Traumato Ortopédica Funcional - NUPEFITO/ISECENSA

(*)e-mail: elizabethvsoares@ outlook.com

Data de recebimento: 23/11/2018 Aceito para publicação: 17/12/2018
} 


\section{INTRODUÇÃO}

No início do desenvolvimento das civilizações, o envelhecimento foi compreendido como uma fase bem característica da vida, não se examinando nenhuma combinação em relação à idade em que se iniciava o envelhecimento. Durante a Idade Média se caracterizava a vida em fases diferentes. Segundo Aristóteles, o início da velhice era a partir de 50 anos, enquanto Santo Agostinho situava 60 anos como idade limite. Hoje no Brasil, segundo a lei $\mathrm{n}^{\circ} 8.842$, entende-se por idoso aquele que tem a idade igual ou superior a 60 anos. Com o passar do tempo, cresce a valorização da produção do trabalho, e o resultado dessa produção para a sociedade. Nos meados do século XX, o olhar ao idoso em relação a sua aposentadoria começou a se modificar, o que antes era marcado como o tempo de descanso e inatividade, agora ganhava importância à maneira como seria vivido, buscando um incentivo maior a uma forma mais saudável e ativa, para que se vivesse o fenômeno do envelhecimento com maior qualidade (JUNIOR, 2006; VIEIRA et al., 2015).

Considerando estimativas para 2020, no Brasil, a população idosa com faixa etária maior que 60 anos será de 28,3 milhões e, para 2050, aproximadamente de 64 milhões. Essa observação gera uma atenção maior a respeito dos fatos incapacitantes e a necessidade, de se efetivar a capacidade funcional do idoso e melhorar sua autonomia e independência (FHON et al., 2012).

O processo progressivo e dinâmico do envelhecimento traz alterações fisiológicas, morfológicas, bioquímicas e psicológicas, acarretando danos e uma maior dificuldade de adaptação. Esse processo complexo resulta da influencia de fatores como: genéticos, estilo de vida e doenças crônicas. Esse acometimento fisiológico do organismo do idoso tem comprometimento importante não só para ele, mas para sua família, para a comunidade e o sistema de saúde, observando que a limitação origina mais vulnerabilidade e dependência, colaborando para uma diminuição da qualidade de vida (VIEIRA et al., 2015; FHON et al., 2012).

Em resposta ao envelhecimento, as alterações do sistema musculoesquelético agem diretamente na funcionalidade, gerando um déficit na mobilidade e independência do idoso. Funções essenciais para a realizações das AVD'S como, força muscular, resistência, equilíbrio estático e dinâmico e amplitude de movimento precisam estar regularmente íntegras para que haja um bom funcionamento biomecânico do corpo aos movimentos solicitados para as atividades. As alterações decorrentes ao envelhecimento, incluem mudanças nos sistemas cardiovascular e musculoesquelético, que uma vez modificados, acabam por diminuir a capacidade ativa do idoso, procedendo em atividades bem próximas ao limiar de desempenho funcional. Com o processo do envelhecimento, aspectos biológicos, doenças e fatores externos podem influenciar o modo em que ele se dá, sendo a queda como um deles (GUCCIONE, 1993; FABRÍCIO et al., 2004).

A série de disfunções generalizadas no organismo do idoso aumenta os riscos de queda, onde torna-se mais vulnerável influenciadas por fatores intrínsecos e extrínsecos. Os fatores determinados como intrínsecos estão ligados a aspectos fisiológicos ou patológicos gerados do processo envelhecimento, já os extrínsecos envolvem as características do ambiente como obstáculos, luminosidade, solo instável etc. $\mathrm{O}$ meio ambiente proporciona certos acontecimentos inesperados que o leva à perda do equilíbrio podendo ocasionar assim uma queda (AVELAR et al., 2016; JUNIOR, 2006; RAMOS, 2005).

Entende-se que as quedas podem causar consequencias de maiores riscos e intensidadecomo: fratura, luxação ou traumatismo cranioencefálico (TCE); e as de menor intensidade: cortes, escoriações e hematomas. As implicações dde uma queda podem promover uma 
deterioração da qualidade de vida do idoso, incluindo a probabilidade de ocasionar a morte. Fatores como a diminuição do equilíbrio, marcha, força muscular e capacidade psicomotora são os mais relevantes quando referente a queda no indivíduo idoso (RAMOS 2005; TALARSKA et al., 2017).

Para que o idoso mantenha sua capacidade de independência é necessário trabalhar para um bom desempenho funcional, pois além da disfunção funcional, há também um menor domínio do equilíbrio. Atualmente aproximadamente $75 \%$ das pessoas a partir dos 70 anos de idade, apresentam limitações do equilíbrio, o que pode ocasionar em uma disfunção local (KAFRI et al., 2017).

Diante do exposto acima, o presente estudo teve como objetivo principal trabalhar a funcionalidade e equilíbrio em idosos, visando aumentar sua qualidade de vida e proporcioná-lo mais autonomia e independência, qualificando o desempenho funcional e a autoconfiança para se equilibrar, dando efetividade a suas atividades básicas da vida diária. Após uma série de avaliações com testes validados, iniciou-se um protocolo com exercícios físicos contando com a utilização de dispositivos ecoeficientes.

\section{METODOLOGIA}

O presente estudo foi de caráter experimental controlado, considerando um levantamento baseado em artigos que pudessem dar embasamento a temas de apoio pertinente a elaboração de um protocolo de treinamento sensório motor. Previamente a sua execução, o projeto de pesquisa foi submetido à avaliação do Comitê de Ética dos Institutos Superiores de Ensino do CENSA (ISECENSA), que aprovou sua realização de acordo com as diretrizes estabelecidas na resolução 196/16 do Conselho Nacional de Saúde segundo certificado de apresentação para apreciação ética sob o número 73624417.06.0000.5524.

A amostra do estudo em pauta foi caracterizada por um $\mathrm{N}=10$ idosas com idade entre $60 \mathrm{e}$ 80 anos divididos em 2 (dois) grupos. Grupo Treinados composto por 6 idosas que realizaram o protocolo de intervenção, e Grupo Não Treinados por 4 idosas que foram submetidas apenas a avaliação pré e pós. Definiu-se uma obrigatoriedade na composição dos grupos em termos de fator de inclusão e exclusão. Os critérios de inclusão foram: indivíduos com idade entre 60 e 80 anos capazes de deambular sem auxílio, hipertensão controlada, que assinaram o Termo de Consentimento Livre e Esclarecido. As idosas portadoras de sequelas neurológicas, com histórico prévio de câncer, arritmia cardíaca não controlada ou que sofreram lesão musculoesquelética recente foram excluídas do estudo.

Em primeiro momento as idosas foram avaliadas através de anamnese e aferição de sinais vitais. Em seguida, foram submetidas a um protocolo de avaliação mais minuciosa através de testes funcionais.

Foram usados os seguintes métodos de avaliação: Escala de equilíbrio Berg (EEB), teste de Up Upgrade e Tempo (TGUGM), e Teste de Alcance Funcional (TAF).

A escala de equilíbrio Berg (EEB) foi usada para avaliar o desempenho do equilíbrio funcional, definir os fatores de risco para dependência e quedas em idosos. Durante a realização deste teste o terapeuta demostra todas as tarefas para o idoso e solicita que o mesmo realize, e a medida que ele necessite de ajuda a pontuação é diminuída. A escala analisa o equilíbrio de acordo 
com 14 itens comuns da vida diária com pontuação máxima de 56 e cada item com pontuação entre 0 (incapaz de realizar a tarefa) e 4 (realiza a tarefa de forma independente); avalia com o indivíduo parado; virando 360 graus; apoio unipodal; levantando-se de uma cadeira, entre outras formas. Para realização do teste é necessário um cronômetro e uma régua. A análise da pontuação é feita da seguinte forma: 0-36 pontos - 100\% risco para quedas, 37-44 pontos- locomoção segura, com recomendações de assistência ou com auxiliares de marcha, 45- 56 pontos- não existe risco de quedas e a locomoção é segura. Além dessa análise pontual, segundo Leonardi (2008), se deve considerar também a amplitude, da pontuação da seguinte forma: 54-56 pontos- cada ponto a menos devemos considerar um risco de quedas de 4\%; 46-54 pontos- cada ponto a menos corresponde 6 a $8 \%$ o risco de quedas; abaixo de 36 pontos- risco de quedas $100 \%$.

Outro instrumento utilizado para avaliação foi o Teste de Up Upgrade e Tempo(TGUGM) que avalia a força dos membros inferiores, coordenação, equilíbrio, juntamente com uma tarefa cognitiva e outras tarefas motoras simultâneas. O TGUGM mede o tempo investido na conclusão da tarefa mencionada e inclui uma avaliação qualitativa $(Q A)$ que permite isolar áreas possíveis de déficit funcional, ajudando-nos a elaborar estratégias específicas de prevenção de queda de acordo com as necessidades dos idosos.

É um teste físico que mede o equilíbrio e a caminhada ao executar uma tarefa cognitiva paralela (dupla tarefa). O teste é subdividido em seis fases que são pontuadas de acordo com o tempo necessário para completar cada fase e o tempo total da prova. Utiliza-se como objetos: duas cadeiras, uma bola, um cone, 8 anéis, um cronômetro e uma trena para marcação dos objetos de acordo com as medidas propostas do teste.

A primeira fase do TGUGM é levantar de forma estável uma cadeira de $42 \mathrm{~cm}$ de altura sem usar as mãos. A segunda fase é chutar uma bola de $19 \mathrm{~cm}$ de diâmetro pesando $0,2 \mathrm{~kg}$ sem perder o equilíbrio e com a maior força possível. A terceira fase é caminhar em direção a um cone localizado a $10 \mathrm{~m}$ da cadeira, enquanto contando de 15 a 0 sem alterar a velocidade de caminhada e sem erros durante a contagem. A quarta fase está passando em torno de um cone, sendo a curva estável o suficiente para não tocar o cone. A fase cinco corresponde à caminhada entre os anéis colocados a $60 \mathrm{~cm}$, onde os participantes observam para ver se eles saem dos anéis. Finalmente, a sexta fase consiste na pessoa sentada novamente na cadeira com um movimento controlado e sem a ajuda de suas mãos. Depois de marcar cada fase do teste, as pontuações são adicionadas em conjunto para obter o resultado total do teste.

Os idosos foram orientados para realizar o teste com as seguintes instruções: "Sente-se com as duas mãos apoiadas no colo. Quando você ouvir Levante-se e vá, levante-se sem usar as mãos; Chute a bola na sua frente o mais forte possível usando o seu melhor pé, então caminhe a um ritmo confortável enquanto você conta para trás de 15 para 0 ao redor do cone sem tocá-lo e volte para a cadeira passando por dentro dos círculos, tentando não para tocar qualquer uma das suas bordas. Finalmente, sente-se novamente, tentando não usar as mãos ".

O primeiro ponto de tempo (T1) correspondeu ao tempo necessário para sentar-se na cadeira; o segundo ponto do tempo (T2) foi o tempo de espera para quando a bola passar pela linha de $8 \mathrm{~m}$; o terceiro ponto de tempo (T3) foi o tempo entre chutar a bola e caminhar em direção ao cone, contando de 15 a 0; O quarto ponto (T4) passou em torno de um cone, sendo a curva estável o suficiente para não tocar o cone; o quinto ponto (T5) correspondeu à caminhada entre os anéis colocados a $60 \mathrm{~cm}$, onde os avaliadores observam para ver se os participantes saem dos anéis. 
Finalmente, o sexto ponto (T6) consistiu quando a pessoa se sentou de volta na cadeira, e o cronômetro finalmente foi parado para registrar o tempo total (TT) do teste.

O desempenho de cada fase do teste foi avaliado em paralelo por um segundo avaliador usando o QA, sendo 0 uma execução deficiente e 3 sendo uma excelente execução. Foi dada uma pontuação total a todos os participantes, indicando a importância do resultado. Índices menores que 12 indicam um alto risco de queda. Foi realizada três tentativas antes da atividade pontuada.

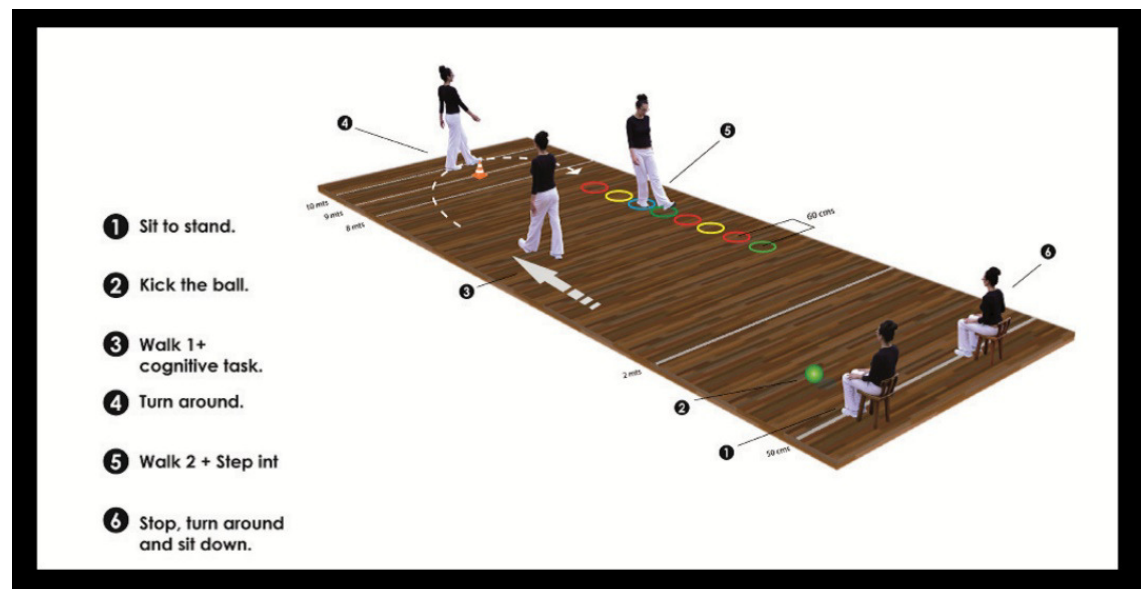

Figura 1: Modo de realização do TGUGM (MORA,2017)

Algumas adaptações do teste foram feitas, como a exclusão da avaliação do cognitivo e do tempo, inclusão de três cones para avaliar mobilidade lateral (dissociação de MMII), um steppra avaliar força de quadril e equilíbrio, pela condição do paciente realizar apoio unipodal (fase de balanço da marcha). Foi feita análise do qualitativo de cada fase do teste, que foram alteradas da seguinte maneira: O quarto ponto (T4) passou em torno dos cones (3), o sexto ponto (T6) consistiu quando a pessoa subiu no step, desceu e sentou de volta na cadeira.

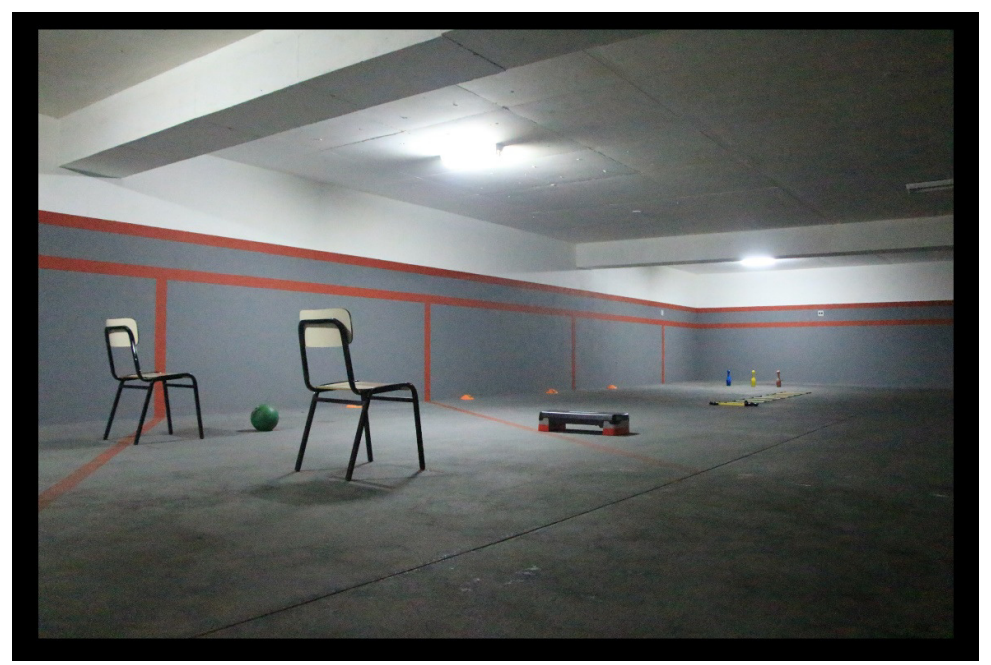

Figura 2: Adaptação do Teste de Up Upgrade e Tempo 
O Teste de Alcance Funcional Anterior (TAF) foi utilizado para avaliar o risco de quedas através da capacidade do idoso em deslocar-se dentro do padrão limite de estabilidade anterior.

Uma fita métrica foi fixada horizontalmente na parede de acordo com a altura do acrômio do participante e paralela ao solo. O idoso é orientado a ficar em pé, paralelo e perpendicularmente em relação à parede e próximo ao início da fita métrica.

Com punhos em posição neutra, cotovelos estendidos e ombro com flexão de $90^{\circ}$, o voluntário é instruído a realizar a inclinação para frente e, em seguida, deve-se verificar o deslocamento sobre ela. A resposta do teste é revelado pela média, depois de três tentativas, a diferença entre a medida na posição inicial e a final registrada na régua. Desvios menores que $15 \mathrm{~cm}$ apontam vulnerabilidade e risco de quedas (KARUKA et., 2011).

Após a valiação inicial com os testes acima, as participantes foram direcionadas à um treinamento sensório motor objetivando melhora da capacidade funcional e equilíbrio com evolução gradual da resistência aplicada. O protocolo constituiu-se de 10 sessões, 3x vezes/semana com duração de 40 minutos por 30 dias.

A análise do estudo e o tratamento foram realizados na Clínica Escola Maria Auxiliadora (CEMA) dos Institutos Superiores de Ensino do CENSA (ISECENSA) e na Creche Escola Baleeira. O local das avaliações tinha como critério ser em ambiente fechado, com ausência de ruídos e com piso liso. Para execução do treinamento, os indivíduos deveriam obrigatoriamente estar com níveis de PA controlado, através de aferição antecipadamente aos exercícios.

Dos dispositivos utilizados para o treinamento sensório motor, 95\% foram confeccionados a partir de materiais recicláveis, como cones de garrafas pet ou papelão; cabos de vassouras personalizados com tinta e fita colorida simulando bastão; escadinha de agilidade preparada a partir de tecido, espuma e velcro; caneleiras de tecido jeans, velcro e areia; kettlebell adaptado feito de tecido, espuma e areia, tapete proprioceptivo produzido com esponjas e papelão; bolas; obstáculos confeccionados a partir de papelão e cabos de vassouras.

O protocolo do treinamento sensório motor foi composto pelos seguintes exercícios: alongamento dos músculos de MMSS e MMII; Aquecimento articular realizando movimentos rotacionais de ombro; flexão e extensão de ombro, cotovelo, punho, dissociação de MMSS com zigzag (feito em dupla), dorsiflexão e plantiflexão de tornozelo; Cinesioterapia resistida para membro superior, com bastão e kettlebell adaptado, aumentando a carga progressivamente: da $1^{\mathrm{a}}$ à $5^{\mathrm{a}}$ sessão $-1 \mathrm{~kg}$ com 2 séries de 10 repetições, e da $5^{\mathrm{a}}$ à $10^{\mathrm{a}}$ sessão $-2 \mathrm{~kg}$, com 3 séries de 12 repetições, sendo as 3 últimas com 3 séries de 15 repetições. Os movimentos realizados foram flexão e extensão de ombro; flexão e extensão de cotovelo; Cinesioterapia resistida para membro inferior com caneleiras, com carga progressiva: da $1^{\text {a }}$ à $5^{\text {a }}$ sessão $-1 \mathrm{~kg}$, com 3 séries de 12 repetições, da $5^{\mathrm{a}}$ à $10^{\mathrm{a}}$ sessão $-2 \mathrm{~kg}$, com 3 séries de 15 repetições. Os movimentos foram de flexão de CF com flexão de joelho a $90^{\circ}$, abdução e adução de coxofemoral, flexão de joelho (paciente em pé). Com o paciente sentado, foram realizados os movimentos de: extensão de joelho com caneleira, e adução com bola. O movimento de plantiflexão foi feito em cadeia cinemática fechada, com apoio dos MMSS na parede; Agachamento em dupla - 5x; Treino lúdico com bola -2 grupos em fila passando a bola pra trás trabalhando mobilidade de MMSS além de incentivar a competitividade entre os grupos; Treino lúdico em roda - jogando a bola e realiza movimento de MI; Treino de sentar e levantar da cadeira -3 séries de 15 repetições; Treino de transferência de cadeira - da $1^{\mathrm{a}}$ à $5^{\mathrm{a}}$ sessão $-1 \mathrm{x} / 10,6^{\mathrm{a}}$ à $10^{\mathrm{a}}$ sessão $2 \mathrm{x} / 10$; Treino global com bola - Paciente em pé de frente para 
parede, com bola na mão, realizando flexão de ombro jogando a bola acima de $90^{\circ}$, após isto ele realiza extensão de CF (realizando agachamento) e então segura a bola voltando a posição inicial 1 série de 10 repetições; Treino de apoio unipodal - segurando em um bastão com flexão de CF e joelho a $90^{\circ}$; Treino de marcha anterior e lateral passando por uma linha de 5 metros (2x); Treino de passada anterior e lateral na escadinha de agilidade $2 \mathrm{X}$; Treino de pegar objeto no chão; Circuito para marcha e equilíbrio, passando por obstáculos com diferentes níveis de altura, step, tapetes proprioceptivos, cones e escadinha de agilidade.

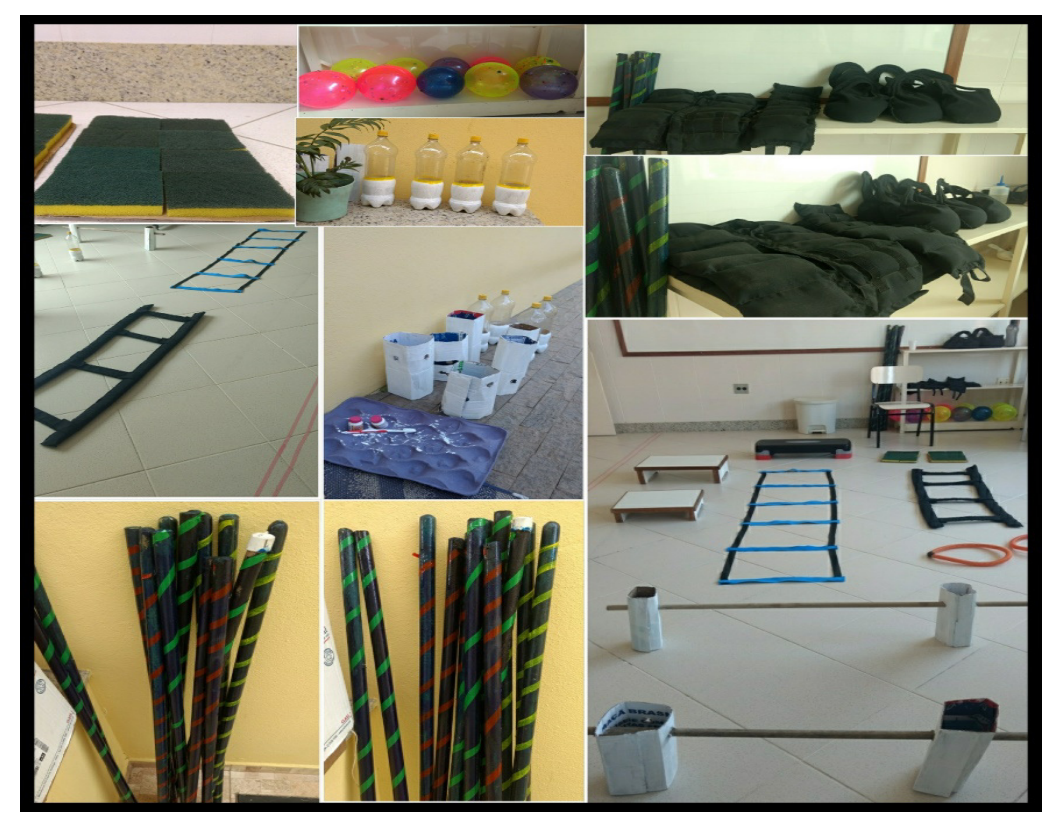

Figura 3: Instrumentação

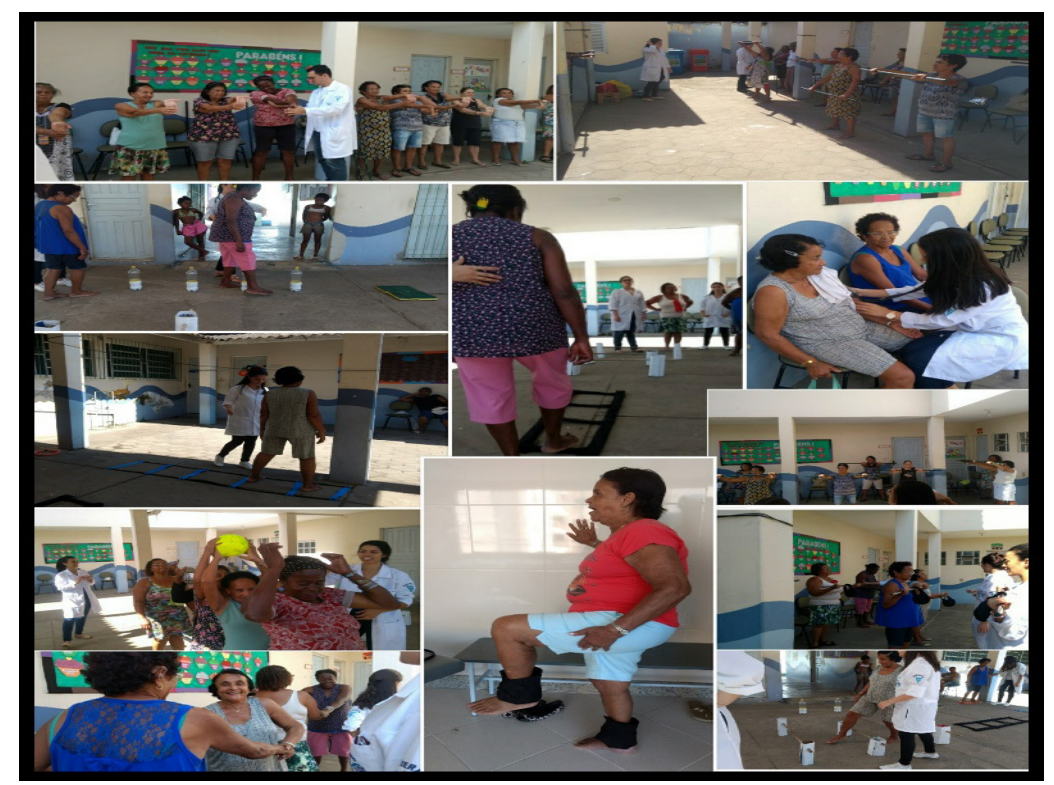

Figura 4: Protocolo de intervenção. 
Após as 10 sessões de treinamento todos os voluntários (grupo Treinados e grupo Não Treinados) foram reavaliados com o mesmo protocolo inicial de avaliação.

A análise dos dados foi feita através de estatística descritiva para cálculo das médias e desvio padrão dos dados coletados. Desta forma, para a verificação de mudanças nos grupos pré e pós-intervenção, foi utilizado o teste t pareado para amostras que apresentaram distribuição normal. O nível de significância para este estudo foi de 0,05 (5\%). As análises foram feitas através de software Windows Office Excel 2010 e GraphPad Prism 7.

\section{RESULTADOS}

O estudo teve como amostra 10 idosas com idade média de 72 $\pm 5,6$; peso $72,8 \pm 12,3$; altura $151,7 \pm 7,1$ e IMC de $32 \pm 7,2$ no grupo Treinados (composto por 6 idosas). A análise das características das idosas que compõem o grupo Não Treinados mostrou uma idade média 71,8 \pm 7 ; peso $61 \pm 10,6$; altura $148,5 \pm 5,8$ e IMC $27,5 \pm 14$. Os dados das características descritas pela média acima, estão representados a seguir na Tabela 1.

Tabela 1: Características médias presentes na amostra (Treinados e Não treinados) com desvio padrão.

\begin{tabular}{ccc}
\hline Variáveis & Treinados(6) & Não \\
Treinados(4) \\
\hline Sexo, F (\%) & $6(100 \%)$ & $4(100 \%)$ \\
Idade (anos), Média (DP) & $72 \pm 5,6$ & $71,8 \pm 7$ \\
Altura (m), Média (DP) & $151,7 \pm 7,1$ & $148,5 \pm 5,8$ \\
Massa (Kg), Média (DP) & $72,8 \pm 12,3$ & $61 \pm 10,6$ \\
IMC (Kg/m²), Média (DP) & $32 \pm 7,2$ & $27,5 \pm 14$ \\
\hline
\end{tabular}

A análise da Escala de Berg demonstrada na figura 5 observou-se que houve diferença estatística entre o grupo Treinados no momento pós treinamento sensório motor sendo $\mathrm{p}<0.05$ quando comparado com o grupo Não Treinados. A média alcançada foi de 46,8 $\pm 6,5$ no grupo Não Treinados e de 53,2 $\pm 2,3$ no grupo Treinados. 


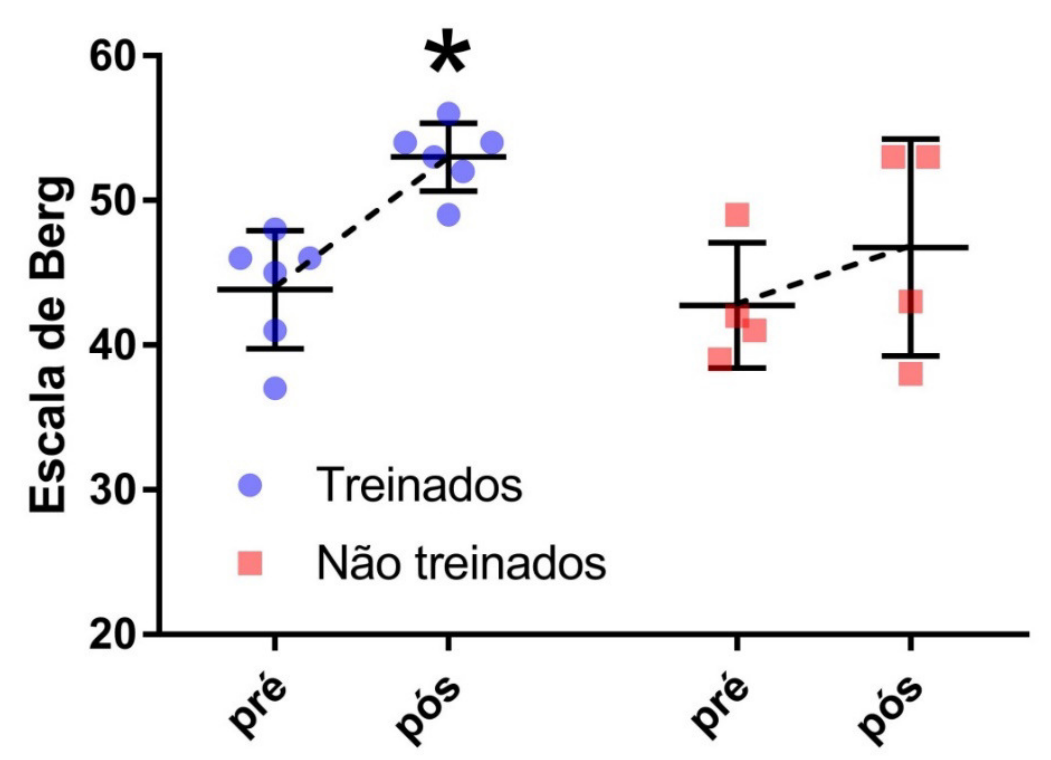

Figura 5: Escala de equilíbrio de Berg. No grupo experimental e controle (*) indica diferença significativa no momento pré intervenção quando comparado ao momento pós sendo $\mathrm{P}<0.05$.

Dados expostos na forma de média \pm DP.

A escala de Berg aplicada, como bem representa a figura acima, descreve grande evidência de melhora sobre as participantes nos aspectos funcionais relacionados às atividades diárias.

Foi apontado na Escala de equilíbrio de Berg que as participantes correspondentes ao Grupo Treinados no momento pré-intervenção apresentavam uma pontuação média $43,6 \pm 0,3$. Segundo a análise de Berg com esta pontuação, as idosas não apresentavam risco de quedas. Porém, segundo Leonardi et al (2008) análise desta escala deve ser feita também de acordo com a amplitude de pontuação. Sendo assim, analisando a amplitude dos dados acima, conseguiu-se verificar que no momento pré do Grupo Intervenção as idosas apresentavam um risco de quedas de aproximadamente de 6 a 8\%. Já no momento pós esse risco diminui para $4 \%$.

A análise da figura 6 referente ao Teste do Alcance Funcional (TAF), demonstrou que houve diferença estatística entre o grupo Treinados no momento pós-treinamento sensório motor sendo $\mathrm{p}<0.05$ quando comparado com o grupo Não Treinado. A média alcançada no momento pós intervenção do grupo Não Treinados foi de 45,8 \pm 5,4 e de 50,8 \pm 2,6 no grupo Treinados. 


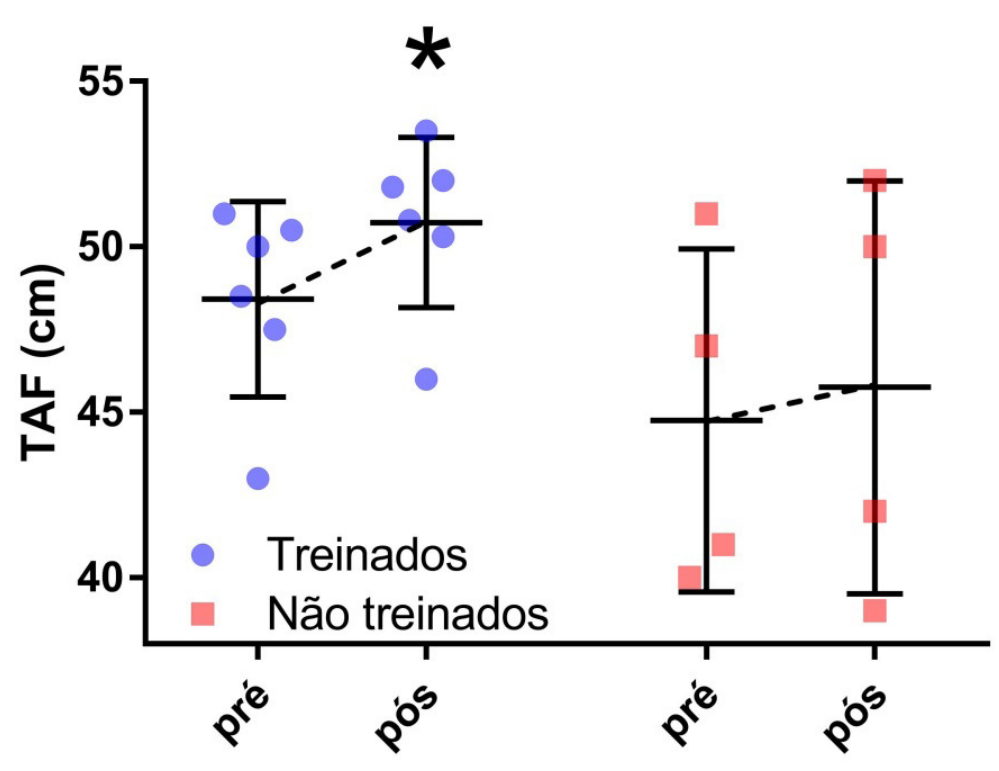

Figura 6. Teste de alcance funcional anterior. No grupo experimental e controle $\left(^{*}\right)$ indica diferença significativa no momento pré intervenção quando comparado ao momento pós sendo $\mathrm{P}<0.05$.

Foi relatado na análise da figura 6 que, as voluntárias do grupo Treinados obtiveram resultados melhores na média geral do teste, concluindo que os dados apontaram que o menor deslocamento anterior medido em cm no período pós-intervenção foi de $46 \mathrm{~cm}$.

Foi observado que houve uma melhora do grupo Treinados com aumento de $3 \mathrm{~cm}$ no momento pós intervenção quando comparado ao momento pré. Analisando o grupo Não Treinados foi verificado que não obtiveram melhora significante.

Os resultados apresentados na figura 7 com relação a avaliação qualitativa (QA) pré e pós do TGUGM observou-se que houve diferença estatística entre o grupo Treinados no momento póstreinamento sensório motor sendo $\mathrm{p}<0.05$ quando comparado com o grupo Não Treinado. A média alcançada no momento pré intervenção no grupo Não Treinados foi de 7,8 \pm 1,3 mantendo-se também no momento pós-intervenção. A análise desta variável no grupo Treinados no momento pré-intervenção foi de $7,8 \pm 1,3$ e no pós intervenção foi de $10,8 \pm 4,9$. 


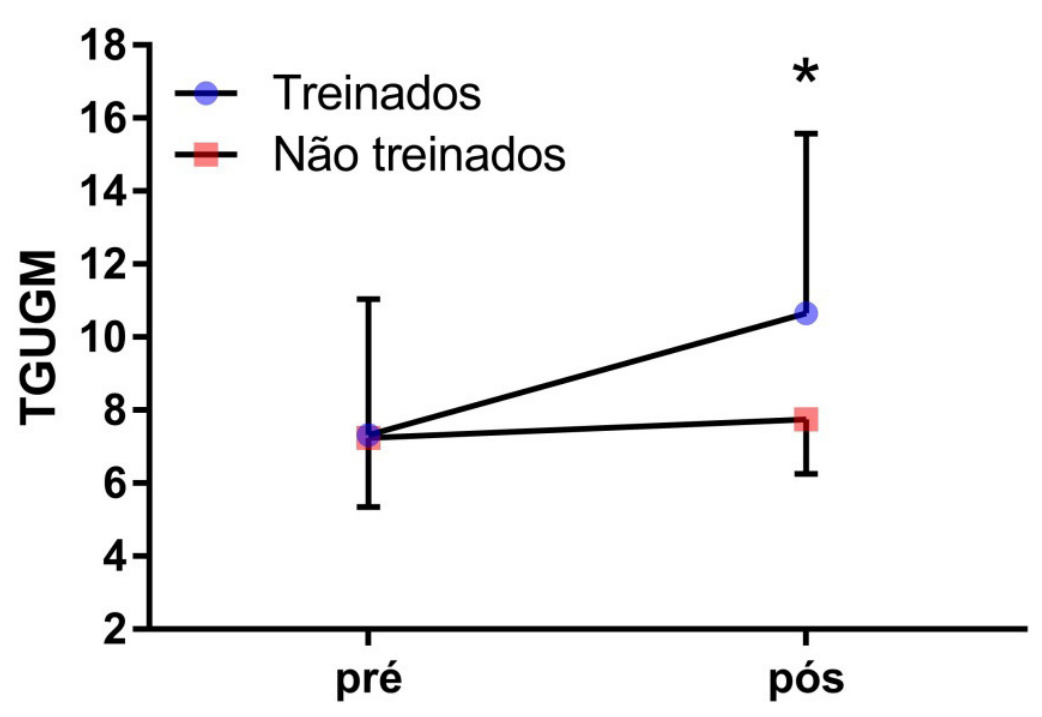

Figura 7: Teste de Up Upgrade e Tempo (TGUGM) no grupo experimental e controle (*) indica diferença significativa no momento pré intervenção quando comparado ao momento pós sendo $\mathrm{P}<0.05$.

A análise qualitativa (QA) do Teste TGUGM de acordo com Mora (2017) é de 12 pontos, verificando-se que valores menores indicam alto risco de quedas. A avaliação dos dados acima verificou melhora com diferença estatística para as idosas do grupo Treinados nos momentos pré e pós, porém a média ficou em torno de 10 pontos, indicando que mesmo assim, as idosas ainda apresentam um risco de quedas. Porém verificou-se durante a reavaliação que houve uma melhora da execução do movimento, como por exemplo, o não uso das mãos para sentar e levantar, melhor mobilidade e equilíbrio para passar entre obstáculos e qualidade de marcha.

\section{DISCUSSÃO}

Após a apresentação dos resultados, inicia-se agora o esclarecimento das relações de causa e efeito, que podem ter gerado diferença nos resultados dos testes, qualidade do movimento e o equilíbrio das idosas participantes, de maneira a comparar o período pré e pós-treinamento sensório motor. Analisar e entender quais as influências que estes resultados aqui presentes, poderão acrescentar para a comunidade Científica.

Os benefícios decorrentes a prática de atividade física regular em indivíduos idosos têm sido alvo de pesquisas por toda a comunidade cientifíca, em virtude da grande relevância deste tema para a população geriátrica.

Avelar et al., (2016) realizou um estudo quase-experimental incluindo 35 idosas aplicado a um tempo de intervenção de 12 semanas, mantendo um período de $50 \mathrm{~min}, 2$ vezes por semana. Os participantes foram divididos em grupo intervenção e controle, onde o grupo intervenção foi submetido ao protocolo de Exercícios de Balanço $(B E C)$ e os indivíduos do controle foram orientados a não praticarem nenhum tipo de atividade. $O$ protocolo de $B E C$ baseado nas atividades diárias é composto por um circuito de exercícios que envolvem equilíbrio estático e dinâmico, passo 
para frente e para trás, marcha larga-posição, alcance funcional, equilíbrio em superfícies instáveis, em apoio unipodal entre outros. Os resultados apresentaram respostas positivas em relação ao $B E C$ melhorando força e potência de MMII, equilíbrio estático, dinâmico e mobilidade. O grupo controle não apresentou qualquer melhora em nenhum nos resultados. No presente estudo foi realizado um protocolo de exercícios terapêuticos incluindo um trabalho proprioceptivo e intervenção sensorial, com duração de 30 dias contabilizando 10 sessões, 3 vezes/ semana com finalidade de melhorar qualidade funcional e equilíbrio. Confirmado em um momento pós intervenção feito por testes validados houve aumento nas pontuações, como demonstrado na figura 5 pela Escala de Equilíbrio de Berg, onde os indivíduos treinados tiveram um acréscimo de 10 pontos mantendo uma média de $53,2 \pm 2,3$ quando comparado ao grupo Não Treinados que não teve diferença significante mantendo média 46,8 $\pm 6,5$ tendo um resultado estatisticamente relevante sendo $p<0.05$.

Segundo Fhon et al. (2012) em um estudo transversal, com predominância do sexo feminino, utilizando questionários de perfil social, histórico de quedas, independência funcional adotando um nível de significância de 0,05 apontou que $50 \%$ dos fatores intrínsecos que causam queda no idoso é referente a alteração do equilíbrio, $30 \%$ refere-se a fraqueza muscular e $25 \%$ dificuldade para marcha. $\mathrm{O}$ presente estudo concentrou-se em exercícios que trabalhassem funcionalidade e equilíbrio, como por exemplo, a passagem por obstáculos de diferentes alturas onde se realizava uma mobilidade articular de quadril, exigindo sinergia de músculos posteriores e anteriores de MI, ao mesmo tempo em que era necessário a estabilização de quadril pelo apoio unipodal momentâneo, promovendo uma melhora da força muscular nos músculos glúteo máximo e médio que são de extrema importância na marcha. Semelhante a essa atividade, a passada lateral na escada de agilidade, recrutava cadeia lateral exigindo equilíbrio. Esses respectivos exercícios agem estimulando à produção de liquido sinovial para lubrificação articular, ainda mais necessária no indivíduo idoso, recrutam musculatura responsável pela estabilização da marcha trabalhando-se em conjunto com agilidade e propriocepção. Essas atividades trabalham habilidades que são necessárias para se obter e manter um bom desempenho funcional incluindo o equilíbrio e trabalhando assim para diminuir fatores de risco preocupantes para queda que estão fortemente presentes quando não observados e tratados.

Na avaliação qualitativa relacionada ao T5 e T6 da adaptação do Teste de Up Upgrade e Tempo (TGUGM), utilizada no estudo aqui descrito, corresponde respectivamente à passada na escada de agilidade e a subida e descida em degrau, mostrando no momento pós-intervenção que as idosas do grupo Intervenção apresentaram melhora nos quesitos de força muscular, equilíbrio e propriocepção.

Diante da literatura estudada, os autores enfatizam a importância da utilização da Escala de Equilíbrio de Berg para identificar a susceptibilidade do idoso a um risco de queda e dependência. A pontuação é fundamentada a partir do tempo em que uma posição pode ser sustentada, no tempo para concluir a função entre outros aspectos de movimentações normais do dia a dia, como por exemplo, a qualidade do movimento de sentar e levantar, transferência de peso de uma cadeira para a outra. Sendo assim, é de extrema importância o uso de testes funcionais para que se tenha direcionamento ao montar o protocolo de tratamento de acordo com os riscos que o indivíduo apresenta. A utilização Escala de Berg no presente estudo referente ao momento pós treinamento apontou que as participantes do grupo Intervenção ultrapassaram suas pontuações, saindo de uma mínima de 37 pontos correspondente a um alto risco de queda para uma pontuação mínima de 49 pontos que corresponde a baixa probabilidade de sofrer queda (MIYAMOTOet al., 2004; KARUKA et al., 2011; SILVA et al., 2017). 
Os resultados apontados confirmam os levantamentos de Fernandes et al., (2012) que estabeleceu um programa de exercícios terapêuticos com objetivo de melhorar equilíbrio, a propriocepção e a marcha em indivíduos sedentários com idade média de 78,1 $\pm 9,3$ anos, consistindo em 12 sessões de 90 minutos. O protocolo consistia em: aquecimento; caminhada; alongamento global; exercícios multisensoriais: treinamento de marcha, propriocepção, força e equilíbrio incluindo uma avaliação pré e pós-intervenção. $\mathrm{O}$ treinamento podia ser feito individual ou em grupo dependendo da progressão do participante. Cabe ressaltar que os testes validados usados na avaliação da pesquisa citada foi diferente do atual estudo. O método de treinamento usado pelos autores é de semelhança ao presente estudo e também obteve resultados satisfatórios em relação à qualidade de vida e redução dos fatores de risco para queda. Por outro lado, a presença do grupo Não Treinados no presente estudo mostrou uma significância maior. Quando analisado os dois grupos em momento pós-intervenção pôde se comparar a mudança na qualidade da execução dos testes, como relatado na Escala de Berg o grupo controle saindo de uma média de 42,8 $\pm 3,7$ alcançou 46,8 $\pm 6,5$ quanto ao grupo intervenção saiu de 43,6 $\pm 4,0$ indo para uma média de 53,2 \pm 2,3 .

Notou-se que as idosas no momento pré intervenção realizavam as atividades solicitadas pelos testes com um desempenho funcional baixo, recebendo notas/pontos de uma realização deficiente, confirmando que o sistema sensorial e o de controle postural são comprometidos com o envelhecimento gerando fatores de risco para a dependência do idoso. Santos et al. (2015) em uma revisão sistematizada justificou a necessidade de empregar exercícios proprioceptivos para sujeitos idosos devido ao um declínio da propriocepção.

Utilizamos o Teste de Alcance Funcional (TAF) que aponta a capacidade de deslocamento anterior do idoso. A importância dessa medição, menciona sua fragilidade de locomoção, mostrando o quanto de vulnerabilidade que o idoso pode ter em se deslocar, como, por exemplo, ao movimento de pegar um objeto a frente. Karuka et al. (2011) afirma que idosos com alcance funcional menor ou igual a $17 \mathrm{~cm}$, apresentam até 13 vezes mais chances de cair. As participantes submetidas ao protocolo mostraram na reavaliação do TAF no momento pós-intervenção um deslocamento anterior mínimo de média $50,8 \pm 2,6$ quando comparado ao grupo treinado que apresentou média de 45,8 \pm 5,4 confirmando assim a eficácia do trabalho sensório motor realizado, destacando a importância de se realizar um protocolo de adequado às fragilidades ocasionadas pelo envelhecimento.

No presente estudo foi apontado que as idosas participantes obtiveram melhora em todos os testes validados, visto pela análise do comparativo pré e pós-treinamento. As voluntárias relataram que todo protocolo de atividades estava sendo eficaz para a melhora do desempenho funcional durante as tarefas diárias, como, movimentos de alcance e aumento da resistência muscular auxiliando no equilíbrio, marcha e o funcionamento físico.

A participação regular na prática de exercícios físicos diminui os efeitos deletérios nos diferentes sistemas do organismo relacionados ao processo fisiológico do envelhecimento, controlando e prevenindo patologias crônico degenerativas (cardiovasculares, diabetes, câncer, hipertensão arterial sistêmica etc) que geram um declínio funcional. Estudos afirmam que desde 1996, o sedentarismo começou a ser analisado como importante fator de risco para patologias degenerativas, além de proporcionar um aumento no risco de queda. Portanto é necessário ser designado um protocolo de exercícios que tenha por objetivo principal diminuir os fatores de risco (CARDOSO et al., 2008; NETO, 2015). 
Mora, (2017) apresenta a utilização do Teste de Up Upgrade e Tempo (TGUGM) para avaliação da marcha e especificamente equilíbrio dinâmico, permitindo verificação das prováveis zonas de déficit funcional em sua avaliação qualitativa. No presente estudo foi feita uma adaptação do TGUGM acrescentando 2 cones para análise de mobilidade lateral e equilíbrio (T5), um step para avaliar capacidade do indivíduo subir um degrau (T6) e ter que realizar apoio unipodal. Os exercícios terapêuticos agiram com objetivos de acordo com a carência dos indivíduos. No momento pré o grupo treinados teve média de 7,4 $\pm 3,7$ e no momento pós $10,8 \pm 4,9$.

Comparando a um estudo de longa duração com treinamento de carga em idosos do gênero masculino apontou-se melhora na capacidade de realizar as AVD's em 72 sessões, porém a análise foi realizada somente pós treinamento e intergrupo. Foi feita dicotomia em 2 grupos, sendo o primeiro grupo, experimental e o segundo, controle. $\mathrm{O}$ grupo experimental realizou atividade $3 \mathrm{x}$ por semana, iniciando com aquecimento por 10 minutos seguido de alongamento, e treinamento de carga $80 \%$ de 1 RM com 2 séries de 8 repetições, intervalo de descanso de 1 minuto e 30 segundos, e de 3 minutos entre cada aparelho, nos exercícios de Leg Press, quadríceps femoral; Leg Curl, isquiotibiais; Vertical Traction, tronco e membro superior recrutando grande dorsal e bíceps secundariamente; Chest Press, tronco e tríceps; Abdominal Crunch, abdominal e Lower back, paravertebral. Já o grupo 2, foi submetido a um treinamento 1x por semana sem carga utilizando os mesmos exercícios, protocolo de séries, intervalo de descanso e por um mesmo período de tempo. O resultado não mostrou significância estatística na escala de BERG, mostrando que o treinamento realizado teve como fim fortalecimento muscular, influenciando na resistência física. Comparando ao presente estudo mostra-se relevância exercitar em conjunto a funcionalidade, com propósito preventivo reduzindo os fatores de risco que leva este indivíduo sofrer quedas (SILVA et al., 2017). Além de obter melhora da capacidade funcional das voluntárias foram utilizados dispositivos ecoeficientes através de materiais recicláveis, corroborando em benefícios para esta população, e estabelecendo eficácia desses materiais sobre o meio ambiente.

Os resultados apresentados no estudo aqui descrito demonstram que um trabalho sensório motor aplicado em apenas 10 sessões foi suficiente para aumentar força muscular e melhorar o equilíbrio, diminuindo o risco de queda deixando o idoso em uma condição mais segura e menos frágil, além de aumentar a eficiência das AVD's, como apontado pelos testes validados no momento pós-intervenção.

O estado de saúde física e mental em idosos depende da interferência de diversos fatores. Estudos ressaltam a importância da relação da condição socioeconômica, apontando indivíduos residentes em locais de cobertura social preocupante com maiores fatores de risco, como por exemplo: higiene precária, violência urbana e carência de serviços de saúde (LIMA e DELGADO, 2010).Considerando o estudo citado, observa-se a necessidade de atenção para tipo de intervenção que venha aumentar a qualidade de vida desses idosos, sendo de fácil realização e acesso para os mesmos. O presente estudo foi elaborado nessa necessidade de acordo com o nível socioeconômico dos participantes, para que fosse algo de baixo custo e de funcionamento efetivo. Sendo assim, utilizou-se dispositivos ecoeficientes através de materiais recicláveis, algo ainda não relatado na literatura. Esses dispositivos foram confeccionados a partir de papelão, cabos de vassouras, esponjas, tecido, areia, velcro, espuma e tinta, os dispositivos, onde todos produzidos pelos terapeutas, do estudo aqui descrito, e por uma costureira voluntária. Utilizou-se material auxiliar como balança para verificar o peso $(1 \mathrm{~kg}-1,200 \mathrm{~kg}$ de areia) para a cada caneleira e kettlebell adaptado. Portanto, ressalta-se a relevância desses dispositivos recicláveis para o meio ambiente e para a fisioterapia quando comparado a materiais convencionais. 
O aumento populacional desordenado, junto com o consumo e com a redução da utilidade dos produtos, traz como consequência resíduos sólidos, o que degrada o meio ambiente tornando-se um dos principais problemas atuais, especialmente quando se nomeia a disposição final desses resíduos. A disposição final dos resíduos, quando não é feita de forma correta, acaba gerando a poluição do ar, solo e água, o que é de grande preocupação (PINHEIRO e FRANCHISCHETTO, 2016).

O crescimento da produção de lixo populacional traz grandes preocupações que atinge a todos os públicos na sociedade atual. O não saber como despejar ou reutilizar esses resíduos é motivo de atenção e acaba sendo um problema de saúde pública. Considerando os aspectos de impacto ambiental e o alto o custo de materiais necessários para a realização dos exercícios, foi elaborado a confecção e utilização de dispositivos por meio de materiais recicláveis e de baixo custo que fossem contra aos fatores negativos da aquisição dos mesmos, e que pudessem ser de fácil acesso para a população mencionada, além de ensiná-los a produzirem seus próprios materiais dando continuidade a execução dos exercícios em âmbito domiciliar, tendo em vista a possível continuação de programas com o mesmo objetivo do presente estudo.

\section{CONCLUSÕES}

De acordo com o desenho metodológico aplicado ao presente estudo, conclui-se que o treinamento sensório motor direcionado ao indivíduo idoso realizado durante dez sessões, determinou um resultado positivo em relação à funcionalidade e equilíbrio estático e dinâmico, melhorando o desempenho das atividades de vida diária diminuindo assim fatores de risco preocupantes para quedas. Entretanto, estudos apontam que melhores resultados são conquistados com alto tempo de intervenção e regularidade. Torna-se assim importante o seguimento do programa de exercícios com mais voluntários por maior período de tempo, implantando também uma avaliação mais criteriosa mensurando força muscular através de dinamometria, flexibilidade através do Banco de Wells além da avaliação do cognitivo do TGUGM ou através do Mini Mental para ter quantitativo de aumento ou redução dos valores analisados.

\section{REFERÊNCIAS}

AVELAR, B. P. et al. Balance Exercises Circuit improves muscle strength, balance, and functional performance in older women. American Aging Association, v. 38, n. 1, p. 1-11, 2016.

CARDOSO, A. S. A. et al. Fatores influentes na desistência de idosos em um programa de exercício físico. Movimento, v. 14, n. 1, p. 225-239, 2008.

FABríCIO, S. C. C.; RODRIGUES, R. A. P.; COSTA JUNIOR, M. L. DA. Causas e consequiências de quedas de idosos atendidos em hospital público TT - Falls among older adults seen at a São Paulo State public hospital: causes and consequences. Revista de Saúde Pública, v. 38, n. 1, p. 93-99, 2004.

FERNANDES, A. M. B. L. et al. Efeitos da prática de exercício físico sobre o desempenho da marcha e da mobilidade funcional em idosos. Fisioterapia em Movimento, v. 25, n. 4, p. 821$830,2012$.

FHON, JACK R. S. et al. Quedas em idosos e sua relação com a capacidade funcional 1. Revista Latino-Americana de Enfermagem, v. 20, n. 5, p. 1-8, 2012.

GUCCIONE. A. A. Fisioterapia Geriátrica. Rio de Janeiro: Guanabara Koogan S.A, 1993 
JUNIOR, E. Envelhecimento e atividade física: diversos olhares sobre a prevenção de quedas. Niterói: GEF/UFF/ANIMA/Brazilfoundation, 2006.

KAFRI, M. et al. Functional Performance and Balance in the Oldest-Old. Journal of Geriatric Physical Therapy, p. 1, 2017.

KARUKA, A. H.; SILVA, J. A. M. G.; NAVEGA, M. T. Análise da concordância entre instrumentos de avaliação do equilíbrio corporal em idosos. Revista Brasileira de Fisioterapia, v. 15 , n. 6 , p. 460-466, 2011.

LEONARDI, M. M, Lopes, G. J., Bezerra P e Borges, A.P.O.; (2008); "Impacto de Desequilíbrio Estático de Dinâmico no Risco de Quedas em Indivíduos com Ataxia Espinocerebelar"; Artigo; Departamento de Neurologia Clínica e Fisioterapia Neuro-Funcional da Universidade de Franca.

LIMA, A. P. DE; DELGADO, E. I. A melhor idade do Brasil: Aspectos Biopsicossociais decorrentes do processo de envelhecimento. ACTA Brasileira do Movimento Humano, v. 1, n. 2, p. 76-91, 2010.

MIYAMOTO, S. T. et al. Brazilian version of the Berg balance scale. Brazilian Journal of Medical and Biological Research, v. 37, n. 9, p. 1411-1421, 2004.

MORA, M.L.A et al. Association between the fall risk, age and educational level in active adult and older women. Salud, Barranquilla vol.33 no.3 Barranquilla Sep./Dec. 2017

NETO;, J. P.; RASO;, W.; BRITO;, C. A. F. Muscular Em Mulheres Idosas. Revista Brasileira de Medicina e Esporte, v. 21, n. 5, p. 2014-2016, 2015.

PINHEIRO, P. T.; FRANCISCHETTO, G. P. P. A política nacional de resíduos sólidos como de catadores de materiais recicláveis. Derecho y Cambio Social, p. 1-24, 2016.

RAMOS, L.R;NETO, J.T. Geriatria e Gerontologia. São Paulo: Manole Ltda, 2005.

SANTOS, K. et al. Associação entre exercício físico e propriocepção em idosos: uma revisão sistemática. Revista Brasileira de Atividade Física \& Saúde, v. 20, n. 1, p. 17, 2015.

SILVA, A. DA et al. Equilíbrio, coordenação e agilidade de idosos submetidos à prática de exercícios físicos resistidos. Revista Brasileira de Medicina do Esporte, v. 14, n. 2, p. 88-93, 2007.

TALARSKA, D. et al. Is independence of older adults safe considering the risk of falls? BMC Geriatrics, v. 17, n. 1, p. 66, 2017.

VIEIRA, S. C. A. L. et al. A força muscular associada ao processo do envelhecimento. Cadernos de graduação: Ciências Biológicas da Saúde, v. 3, n. 1, p. 93-102, 2015. 